\title{
Kreuth IV: European consensus proposals for treatment of haemophilia with coagulation factor concentrates
}

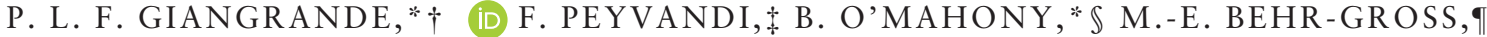 \\ A. HILGER, **W. SCHRAMM $+\dagger$ and P. M. MANNUCCI + \\ *European Haemophilia Consortium, Brussels, Belgium; †University of Oxford, Oxford, UK; $\$$ Angelo Bianchi Bonomi \\ Hemophilia and Thrombosis Centre, Fondazione IRCCS Ca' Granda Ospedale Maggiore Policlinico, Milan, Italy; $\$$ Trinity \\ College, Dublin, Ireland; $\uparrow$ European Directorate for the Quality of Medicines and HealthCare (EDQM), Council of Europe, \\ Strasbourg, France; *Paul-Ehrlich-Institut, Langen; and †Rudolf-Marx-Foundation, University of Munich, Munich, \\ Germany
}

Introduction: This report summarizes recommendations relating to haemophilia therapy arising from discussions among experts from 36 European countries during the 'Kreuth IV' meeting in May 2016. Aim: The objective of the meeting was for experts in the field of haemophilia from across Europe to draft resolutions regarding current issues relating to the treatment of haemophilia. Results: Hospitals providing clinical care for people with haemophilia and related disorders are strongly recommended to seek formal designation as either European Haemophilia Treatment Centres (EHTC) or European Haemophilia Comprehensive Care Centres (EHCCC). There should be agreed national protocols or guidelines on management of the ageing patient with haemophilia. The minimum consumption of factor VIII and IX concentrate in any country should be 4 IU and 0.5 IU per capita of general population respectively. Treatment for hepatitis $\mathrm{C}$ with direct-acting antiviral agents should be provided to all people with haemophilia on a priority basis. Genotype analysis should be offered to all patients with severe haemophilia. Genetic counselling, when given, should encompass the recommendation that genetic relatives of the affected person be advised to seek genetic counselling. People with inhibitors should have access to bypassing agents, immune tolerance and elective surgery. National or regional tenders for factor concentrates are encouraged. Outcome data including health related quality of life should be collected. Treatment with extended half-life factors should be individualized and protection against bleeding should be improved by increasing trough levels. Steps should be taken to understand and minimize the risk of inhibitor development. Conclusion: It is hoped that these recommendations will help to foster equity of haemophilia care throughout Europe.

Keywords: factor IX, factor VIII, guidelines, haemophilia, inhibitors, prophylaxis

\section{Introduction}

This manuscript describes the outcome of deliberations from the latest in a series of meetings devoted to optimal clinical use of blood components and of plasma-derived medicinal products with a focus on the treatment of haemophilia and related disorders in Europe. These meetings have been convened periodically under the joint aegis of the Paul Ehrlich Institut (PEI), the Ludwig-Maximilian-University of Munich

Correspondence: Paul L. F. Giangrande M.D., European Haemophilia Consortium, Rue de l'Industrie, B-1000 Brussels, Belgium.

Tel.: +32 2893 2470; e-mail: paul.giangrande@gtc.ox.ac.uk

Accepted after revision 26 January 2017
(LMU) and the Council of Europe through its European Department for the Quality of Medicines and Healthcare (EDQM). It is therefore important to emphasize that delegates were invited from the 47 member states of the Council of Europe, which covers a much larger territory than the 28 member states of the European Union. Previous meetings in this framework were held in Wildbad Kreuth in southern Germany in 1999, 2009 and 2013 [1]. The principal recommendations which emerged from the last meeting in April 2013 were subsequently endorsed by the Committee of Ministers of the Council of Europe in April 2015 (Resolution CM/Res[2015]3). The conference was an opportunity for nominated delegates from all Council of Europe member and observer states, along with colleagues from academia, the European 
Haemophilia Consortium (EHC) and European Medicines Agency (EMA), to review trends in the use of coagulation factor concentrates. The three areas which were set as objectives for discussion in relation to haemophilia were: critical appraisal of the status $q u o$ and identification of gaps in clinical and outcomes research in haemophilia; perspectives on innovative products and identification of best practice; and future needs in haemophilia care. Participants heard a series of 'state of the art' presentations and then met in workshops to draw up consensus recommendations relating to research and regulatory policies concerning haemophilia therapy in Europe [2]. Data from the latest 2015 survey from the European Haemophilia Consortium (EHC) were presented and discussed [3]. These were very helpful in highlighting both areas where progress has been made as well as other areas where further effort is still required to improve the quality of care. The EHC plans to conduct further surveys on a regular basis in the coming years in order to monitor adoption of these latest recommendations. It is also planned to submit these latest recommendations to the Committee of Ministers of the Council of Europe as high-level political support will add considerable weight to the recommendations.

\section{Objectives}

The aim of the meeting was for experts in the field of haemophilia from across Europe (including patient organization representatives, clinicians and regulators) to draft resolutions regarding current issues relating to the treatment of haemophilia.

\section{Recommendations of the 'Kreuth IV' meeting}

\section{Recommendation 1}

Hospitals providing clinical care for people with haemophilia and related disorders are strongly recommended to seek formal designation as either European Haemophilia Treatment Centres (EHTC) or European Haemophilia Comprehensive Care Centres (EHCCC).

The EUHANET project, funded by the European Commission, has established criteria for the designation of two tiers of haemophilia treatment centres, namely European Haemophilia Treatment Centres (EHTC) and European Haemophilia Comprehensive Care Centres (EHCCC) [4]. The ultimate aim of the project was to standardize the level of care within Europe and, at the same time, ensure that access to comprehensive care and replacement therapy is equitable in all parts of individual countries. Key criteria in the assessment of treatment facilities include the number of patients treated as well as the range of multidisciplinary support and laboratory test available. Applications are reviewed by a panel which includes representatives from both the European Haemophilia Consortium (EHC) and the European Association for Haemophilia and Allied Disorders (EAHAD). Although the system currently relies on self-certification, it is hoped to introduce a system of external audit or accreditation in the future. Participation in the scheme is not mandatory but over 120 centres from 30 countries have already applied for certification through the scheme.

\section{Recommendation 2}

There should be agreed national protocols or guidelines on management of the ageing patient with haemophilia. Treatment centres are encouraged to include an appropriate general physician in the comprehensive care team.

Improvements in the treatment of haemophilia have led to a striking increase in longevity of people with haemophilia in recent years such that life expectancy now approaches that of the general population. In the UK, the number of patients with severe haemophilia aged 60 or more has trebled over the last 40 years [5]. However, the management of elderly people with haemophilia presents new challenges in the form of complex medical problems which many haematologists will not be accustomed to dealing with, such as cardiac and respiratory diseases [6]. Paediatricians have been involved in haemophilia care for decades and a strong feeling emerged at the conference that the time has now come to invite physicians experienced in the care of the elderly to also become part of the multidisciplinary team in haemophilia treatment centres.

\section{Recommendation 3}

The minimum consumption of Factor VIII concentrate in any country should be 4 IU per capita of general population.

A minimum consumption of 3 IU per capita was recommended at the Kreuth III meeting in 2013, on the basis that this was the threshold required to ensure children could be treated on a prophylactic basis [1]. It is encouraging to see in the most recent EHC survey from 2015 that Factor VIII consumption has increased in 22 out of 30 countries since the last survey in 2013. However, national consumption was still below the threshold of 3 IU per capita in 7 countries [3]. Factor VIII consumption ranged from 0.46 IU per capita in Albania to 9.4 IU per capita in Hungary. There has been growing support for prophylactic treatment of adults in the last few years, based on the results of the SPINART and other prospective randomized clinical trials [7,8]. Many clinicians now offer secondary prophylaxis to adults with severe haemophilia, in the light of results from such studies which have provided clear evidence of a benefit in terms of both reduction 
in frequency of bleeds as well improvement in quality of life. In order to encourage and facilitate wider adoption of prophylaxis in adults, the group recommended a further increase in recommended minimum Factor VIII consumption in a country to 4 IU per capita. The group wishes to emphasize that this consumption level is regarded as the minimum acceptable one and a threshold of 4 IU per capita should not be viewed by public health authorities as the optimum level. The group also recommended that data expressed as units per severe patient should also be collected in parallel in future. It was also acknowledged that the use of novel coagulation factor concentrates with extended half-lives will further complicate analysis of concentrate use in terms of IU per capita in the coming years. One solution would be to agree on a conversion factor for each product based on the degree of prolongation.

\section{Recommendation 4}

The minimum consumption of Factor IX concentrate in a country should be 0.5 IU per capita of general population.

Working parties at previous Kreuth meetings have never before issued a recommendation in relation to Factor IX consumption. Previous international surveys have also largely focused on Factor VIII consumption. After consideration of the data from the 2015 EHC survey, the group agreed unanimously to recommend a minimum national Factor IX consumption of $0.5 \mathrm{IU}$ per capita. This was the median value in the most recent EHC survey, with a range of from 0.1 IU per capita in Romania to 2.47 IU per capita in the Republic of Ireland [3]. The lower consumption level of Factor IX (compared to factor VIII) reflects the lower frequency of haemophilia B in the European population.

\section{Recommendation 5}

Treatment for hepatitis $\mathrm{C}$ with direct-acting antiviral agents should be provided to all people with haemophilia on a high priority basis.

Many patients with haemophilia were infected with hepatitis C (HCV) in the 1970s and 80s, before the introduction of virucidal techniques such as heat or solvent/detergent treatment of plasma for fractionation. HCV infection is typically slow to progress, often causing few symptoms until cirrhosis or liver cancer develops after several decades. The EUHASS survey has shown that hepatocellular carcinoma is now the leading malignancy diagnosed in people with haemophilia. Interferon and ribavirin have been used to treat some patients in the past, but only approximately half of them achieved sustained viral eradication with this combination, which was also associated with significant side-effects. The recent development of direct-acting antiviral agents such as sofosbuvir and simeprevir offers a new opportunity to treat patients with haemophilia although the cost of treatment is a barrier. The probability of viral eradication is much higher with these new drugs, which are also less toxic and administered orally for shorter time period. The group felt very strongly that people with haemophilia and related disorders constitute a special group which merits priority for treatment, given the iatrogenic source of infection.

\section{Recommendation 6}

Genotype analysis should be offered to all patients with severe haemophilia. Patients shall be free to decide whether or not to take up this possibility. Genetic counselling of the affected person, when given, should encompass the recommendation that genetic relatives of the affected person be advised to seek genetic counselling.

A recommendation that genetic counselling should be available to families affected by haemophilia was made at the second Kreuth meeting in 2009. No genetic tests are required, for example, to advise the daughter of a man with haemophilia that she will be a carrier of the condition or that the son of an affected man will be free of the condition. However, the situation for many women in the wider kindred is not so clear and can only be established through DNA-based testing using technology which is now widely available. This information can then be used to determine foetal sex early during pregnancy if a women wishes to undergo antenatal diagnosis with a view to termination of an affected pregnancy (in countries where this is permitted by law). Knowledge of the particular genotype responsible for haemophilia in a patient can also be used to give an indication of the risk of inhibitor development. Although patients with a severe phenotype should be prioritized for genetic studies, genotyping is also desirable in cases of mild and moderate haemophilia and for patients with other congenital bleeding disorders.

\section{Recommendation 7}

People with inhibitors should have access to immune tolerance.

A prospective international trial of immune tolerance induction has provided clear evidence that the majority of patients with haemophilia A and inhibitors achieve tolerance: $70 \%$ of patients achieved complete tolerance and a further $5 \%$ achieved a partial response [9]. Although the infusion of high doses of Factor VIII for prolonged periods requires a high financial outlay, this is cost effective in the longer term through the avoidance of repeated treatment of 
bleeding episodes with much more expensive bypassing agents. A recommendation that all patients with inhibitors should be offered immune tolerance was made at the very first Kreuth meeting in 1999. However, the most recent EHC survey also provides evidence that immune tolerance induction therapy is still not readily available in several European countries and so the group felt it important to promulgate this recommendation again.

\section{Recommendation 8}

People with inhibitors should have access to elective surgery at a specialist centre with relevant experience.

It is now accepted that surgery in patients with haemophilia and inhibitors can now be performed safely using bypassing agents [10,11]. Despite the considerable experience and number of publications in this field, it is still that case that patients with inhibitors are denied elective surgery such as arthroplasty which could improve their quality of life [12]. In part, this is because some treatment centres still harbour reservations about the potential for bleeding but it is also recognized that the initial high cost may be a considerable barrier. However, a number of economic evaluations indicate that surgery may prove cost effective in the longer term $[13,14]$. The firm opinion of the group was that surgery in patients with inhibitors should only be carried out in centres with previous experience. Where this is not feasible locally, patients should be referred to another centre with the requisite experience (across national borders if necessary).

\section{Recommendation 9}

National or regional tenders for factor concentrates are encouraged and should always include both haemophilia clinicians and national haemophilia patient representatives.

The cost of coagulation factor concentrates accounts for around $80 \%$ of the overall budget for haemophilia care $[15,16]$. If a budget is limited, then the ability to purchase product at a low price is vital in order to maximize the volume purchased. A recent international survey has demonstrated that tenders are a very effective way to reduce prices through open competition between manufacturers [17]. This study emphasized that the best outcome in terms of price was obtained when both haemophilia physicians as well as patient representatives were engaged in the process. In the UK, the price of recombinant Factor VIII was almost halved after a series of competitive tenders [18]. The process was fully supported by patients in the UK and no increased risk of inhibitor development was observed after product switching [19]. Of course, as already pointed out through the Kreuth III recommendations, cost should not be the sole consideration in product tenders: product safety should always be the primary selection criterion.

\section{Recommendation 10}

Outcome data including health related quality of life should be collected with appropriate study design, e.g. annualized bleed rates (ABR), mortality, joint score and time off from education or employment.

The group recognized that both clinicians and health agencies which purchase products for the treatment of haemophilia face an increasingly difficult task in choosing the most appropriate products at a time when so many novel products become available. Formal health technology assessments play an ever increasing role in product selection. Data on novel products must be collected during routine reviews and be used by clinicians to persuade payers to adopt novel products. Ideally, such data from individual treatment centres should also be collated at national level through a database. Formal clinical trials also have an increasingly important role in providing objective data. The appropriate study design depends on the study question and, for this, the correct terminology needs to be used:

Case series: group of patients on whom cross-sectional data are collected

Register: database that contains information on all inhabitants of a country, or a complete subset of those, i.e. all patients with a certain disorder

Cohort: a group that is followed over time

Randomised trial: a cohort with two arms defined by an intervention, which has been allocated by a random process.

\section{Recommendation 11}

Treatment with extended half-life factors should be individualized and protection against bleeding should be improved by increasing trough levels.

A number of novel coagulation factor concentrates with extended half-lives have been developed, some of which are already licensed in some countries. Perceived advantages of these products include a reduction in the frequency of venepuncture and the attainment of higher trough levels than the current conventional target of $1 \%\left(0.01 \mathrm{IU} \mathrm{ml}^{-1}\right)$. The benefits are particularly evident for longer-acting novel Factor IX products, as these have a 5 -fold increase in half-life as compared to a much more modest 1.5 -fold increase for novel factor VIII products. Many advocate the adoption of higher trough levels of up to $15 \%$ as this would minimize the risk of bleeding and allow patients to lead more active lives [20]. However, the group could not reach consensus on what the desirable minimum trough levels should be with such 
new agents and further research is required in this area. It was recognised that treatment regimens should be individualized, according to the lifestyle and individual pharmacokinetics in each patient. However, treatment regimens should not be dictated by trough and peak levels alone. The primary parameter to guide treatment should be the frequency of bleeds: as recent UK guidelines on the clinical utilization of these novel products emphasize, the goal in children should be an annualized bleeding rate of 0 [21].

\section{Recommendation 12}

There is increasing evidence that the incidence of inhibitors amongst previously untreated patients (PUPs) varies between products. Steps should be taken to understand and minimize this risk.

Now that the reduction of the risk of transmission of pathogens like HIV and hepatitis through plasmaderived medicinal products has effectively been maximized, the development of inhibitors has become the principal complication of haemophilia therapy. The SIPPET study reported a significantly increased risk of inhibitor developments amongst previously untreated patients (PUP) who received recombinant Factor VIII products, when compared to subjects who received plasma-derived products [22]. Other factors which have been implicated in the appearance of inhibitors in PUPs include ethnicity, intensity of early treatment and early prophylaxis [23]. Patients, or parents in the case of young children, should be involved in discussions related to product choice and usage during the early period of treatment. It is now also recognized that a significant proportion of patients with non-severe haemophilia can develop inhibitors after treatment with coagulation factor concentrates. Adults with mild haemophilia who, for example, undergo minor invasive procedures such as dental extractions should be checked for treatment status in relation to coagulation factors. In cases where they are still effectively PUPs and in the vulnerable period of $<50$ exposure days, the use of desmopressin (DDAVP) instead of coagulation factor concentrates will reduce the risk of inhibitor development.

\section{Acknowledgements}

The Kreuth IV initiative was co-sponsored by the Paul Ehrlich Institut (PEI), Langen, Germany, the Ludwig-Maximilian-University (LMU) Munich, Germany and the European Directorate for the Quality of Medicines \& HealthCare (EDQM), Council of Europe, Strasbourg, France. Financial support was also provided by the Rudolf Marx Stiftung in Munich, Germany. No financial support for the meeting was provided by pharmaceutical companies.

\section{Author contributions}

All authors participated in the meeting and related workshops and contributed to the drafting and review of this manuscript.

\begin{abstract}
Disclosures
PG has received speaker and/or consultancy fees from Bayer, Biotest, CSL Behring, LFB, NovoNordisk, Pfizer and Shire. FP has received speaker and/or consultancy fees from Ablynx, Bayer, Grifols, Kedrion, LFB, NovoNordisk, Octapharma and Sobi. She has received funds for research from Ablynx, Alexion, Kedrion and Novonordisk (although all money was paid to the Fondazione Luigi Villa). PM has received honoraria for lectures as speaker or chair symposia organized by Bayer, Grifols, Kedrion, LFB, Novo Nordisk and Pfizer. He also acted as scientific consultant for Bayer, Baxalta and Kedrion. The other authors stated that they had no interest which might be perceived as posing a conflict or bias.
\end{abstract}

\section{References}

1 Giangrande P, Seitz R, Behr-Gross ME et al. Kreuth III: European consensus proposals for treatment of haemophilia with coagulation factor concentrates. Haemophilia 2014; 20: 322-5.

2 Available at https://pyweb.edqm.eu/qualif/ freepub. Accessed December 13, 2016.

3 O'Mahony B, Savini L, O'Hara J, Bok A. Haemophilia care in Europe: a survey of 37 countries [submitted for publication].

4 Giangrande P, Calizzani G, Candura F, Mannucci PM, Makris M, Seitz R. The European standards of Haemophilia Centres. Blood Transfus 2014; 12(Suppl. 3): s525-30.

5 UKHCDO Annual Report 2015 (figure 4, page 15). Available at www.ukhcdo.org. Accessed December 13, 2016.

6 Angelini D, Konkle BA, Sood SL. Aging among persons with hemophilia: contemporary concerns. Semin Hematol 2016; 53: 35-9.

7 Manco-Johnson MJ, Kempton CL, Reding MT et al. Randomized, controlled, parallel-group trial of routine prophylaxi vs. on-demand treatment with sucrose-formulated recombinant factor VIII in adults with severe haemophilia A (SPINART). J Thromb Haemost 2013; 11: 1119-27.

8 Tagliaferri A, Feola G, Molinari AC et al. Benefits of prophylaxis versus ondemand treatment in adolescents and adults with severe haemophilia A: the POTTER study. Thromb Haemost 2015; 114: 35-45.

9 Hay CR, DiMichele DM. The principal results of the International Immune Tolerance Study: a randomized dose comparison. Blood 2012; 119: 1335-44.

10 Santagostino E, Escobar M, Ozelo M et al. Recombinant activated factor VII in the treatment of bleeds and for the prevention of surgery-related bleeding in congenital haemophilia with inhibitors. Blood Rev 2015; 29(Suppl 1): S9-18.

11 Négrier C, Lienhart A, Numerof R et al. SURgical interventions with FEIBA (SURF): international registry of surgery in haemophilia patients with inhibitory antibodies. Haemophilia 2013; 19: e143-50.
12 Dekoven M, Wisniewski T, Petrilla A, Holot N, Lee WC, Cooper D. Patient/caregiver perceived benefits and barriers to elective orthopaedic surgery (EOS) in patients with congenital hemophilia with inhibitors. J Med Econ 2012; 15: 305-12.

13 Lyseng-Williamson KA, Plosker GL. Recombinant factor VIIa (eptacog alfa): a pharmacoeconomic review of its use in haemophilia in patients with inhibitors to clotting factors VIII or IX. Pharmacoeconomics 2007; 25: 1007-29.

14 Ali JM, Howieson AJ, Lawrence K, Chitnavis D, Chitnavis J. Total knee arthroplasty in a patient with haemophilic arthropathy and high-titer inhibitors: a cost analysis. J Arthroplasty 2012; 27: 1413.e11-14.

15 Guh S, Grosse SD, McAlister S, Kessler CM, Soucie JM. Healthcare expenditure for males with haemophilia and employersponsored insurance in the US, 2008. Haemophilia 2012; 18: 268-75.

16 Rocha P, Carvalho M, Lopes M, Araújo F. Costs and utilization of treatment in patients with haemophilia. BMC Health Serv Res 2015; 15: 484. 
17 O’Mahony B, Noone D, Prihodova L. Survey of coagulation factor concentrates tender and procurement procedures in 38 European Countries. Haemophilia 2015; 21: 436-43.

18 Hay CR. Purchasing factor concentrates in the 21st century through competitive tendering. Haemophilia 2013 Sep; 19: 660-7.

19 Hay CR, Palmer BP, Chalmers EA et al. The incidence of factor VIII inhibitors in severe haemophilia A following a major switch from full-length to Bdomain-deleted factor VIII: a prospective cohort comparison. Haemophilia 2015; 21: 219-26.

20 Skinner MW. Haemophilia care-past, present and future from a patient perspective. Haemophilia 2012; 18(Suppl 5): 3-5.

21 Collins P, Chalmers E, Chowdary P et al. The use of enhanced half-life coagulation factor concentrates in routine clinical practice: guidance from UKHCDO. Haemophilia 2016; 22: 487-98.

22 Peyvandi F, Mannucci PM, Garagiola I et al. A randomized trial of factor VIII and neutralizing antibodies in hemophilia A. N Engl J Med 2016; 374: 2054-64.

23 Mannucci PM, Mancuso ME, Franchini M. Tailoring hemostatic therapies to lower inhibitor development in previously untreated patients with severe hemophilia A. J Throm Haemostas 2016; 14: 1330-6. 